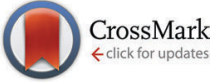

Cite this: Phys. Chem. Chem. Phys., 2016, 18, 24729

Received 28th July 2016, Accepted 17th August 2016

DOI: 10.1039/c6cp05240a

www.rsc.org/pccp

\section{The photochemistry of sodium ion pump rhodopsin observed by watermarked femto- to submillisecond stimulated Raman spectroscopy $\dagger$}

\author{
Yusaku Hontani, $\ddagger^{\mathrm{a}}$ Keiichi Inoue, $\ddagger^{\mathrm{bcd}}$ Miroslav Kloz, ${ }^{a}$ Yoshitaka Kato, ${ }^{b}$ \\ Hideki Kandori ${ }^{b c}$ and John T. M. Kennis*a
}

\begin{abstract}
Krokinobacter rhodopsin 2 (KR2) is a recently discovered light-driven $\mathrm{Na}^{+}$pump that holds significant promise for application as a neural silencer in optogenetics. KR2 transports $\mathrm{Na}^{+}$(in $\mathrm{NaCl}$ solution) or $\mathrm{H}^{+}$ (in larger cation solution, e.g. in $\mathrm{CsCl}$ ) during its photocycle. Here, we investigate the photochemistry of KR2 with the recently developed watermarked, baseline-free femto- to submillisecond transient stimulated Raman spectroscopy (TSRS), which enables us to investigate retinal chromophore dynamics in real time with high spectral resolution over a large time range. We propose a new photocycle from femtoseconds to submilliseconds: J (formed in $\sim 200 \mathrm{fs}) \rightarrow \mathrm{K}(\sim 3 \mathrm{ps}) \rightarrow \mathrm{K} / \mathrm{L}_{1}(\sim 20 \mathrm{ps}) \rightarrow \mathrm{K} / \mathrm{L}_{2}$ $(\sim 30 \mathrm{~ns}) \rightarrow \mathrm{L} / \mathrm{M}(\sim 20 \mu \mathrm{s})$. KR2 binds a $\mathrm{Na}^{+}$ion that is not transported on the extracellular side, of which the function is unclear. We demonstrate with TSRS that for the D102N mutant in $\mathrm{NaCl}$ (with $\mathrm{Na}^{+}$ unbound, $\mathrm{Na}^{+}$transport) and for WT KR2 in $\mathrm{CsCl}$ (with $\mathrm{Na}^{+}$unbound, $\mathrm{H}^{+}$transport), the extracellular $\mathrm{Na}^{+}$ binding significantly influences the intermediate $\mathrm{K} / \mathrm{L} / \mathrm{M}$ state equilibrium on the photocycle, while the identity of the transported ion, $\mathrm{Na}^{+}$or $\mathrm{H}^{+}$, does not affect the photocycle. Our findings will contribute to further elucidation of the molecular mechanisms of KR2.
\end{abstract}

Krokinobacter rhodopsin 2 (KR2) is the first identified outward sodium ion $\left(\mathrm{Na}^{+}\right)$pump rhodopsin (NaR) reported in 2013. ${ }^{1}$ KR2 is composed of seven trans-membrane helices (TM1-TM7), and covalently binds a common chromophore, all-trans retinal, which undergoes isomerization to the 13-cis form upon photon absorption. With the unique ability of the unidirectional $\mathrm{Na}^{+}$ pump, KR2 is of great interest in optogenetic applications as a neural silencer. ${ }^{2,3}$ The crystal structures of the pentameric state of KR2 were solved by Gushchin et al. in $2015,{ }^{4}$ which showed that $\mathrm{Na}^{+}$binding exists on the extracellular side of the interfaces between two different protomers. This binding site consists of Tyr25 (on TM1), Thr87 and main chain carbonyl groups of Thr83 and Phe86 (on TM2) of the same protomer, Asp102 (on the loop between TM2 and TM3) of the neighboring protomer

\footnotetext{
${ }^{a}$ Department of Physics and Astronomy, Faculty of Sciences, VU University Amsterdam, De Boelelaan 1081, 1081 HV Amsterdam, The Netherlands. E-mail: j.t.m.kennis@vu.nl

${ }^{b}$ Department of Frontier Materials, Nagoya Institute of Technology, Showa-ku, Nagoya 466-8555, Japan

${ }^{c}$ OptoBioTechnology Research Center, Nagoya Institute of Technology, Showa-ku, Nagoya 466-8555, Japan

${ }^{d}$ PRESTO, Japan Science and Technology Agency, 4-1-8 Honcho, Kawaguchi, Saitama 332-0012, Japan

$\dagger$ Electronic supplementary information (ESI) available. See DOI: 10.1039/c6cp05240a

$\$$ These authors contributed equally to this work.
}

and two water molecules (Fig. 1a). Importantly, the $\mathrm{Na}^{+}$ions on this binding site are not the ones that are transported. ${ }^{1,4}$ Moreover, it was reported that the D102N mutant of KR2 does not bind $\mathrm{Na}^{+}$ on the extracellular binding site, but still pumps $\mathrm{Na}^{+}$as well as WT KR2. ${ }^{2} \mathrm{KR} 2$ also pumps $\mathrm{Li}^{+}$, but transports only $\mathrm{H}^{+}$in the absence of $\mathrm{Na}^{+}$and $\mathrm{Li}^{+}$, e.g. in solution containing only larger cations such as $\mathrm{K}^{+}, \mathrm{Rb}^{+}$and $\mathrm{Cs}^{+}{ }^{+1,2}$ Attenuated total reflection-Fourier transform infrared (ATR-FTIR) studies showed $\mathrm{K}^{+}, \mathrm{Rb}^{+}$and $\mathrm{Cs}^{+}$do not bind to the ion binding site on the extracellular side of KR2.,

A femtosecond transient absorption study indicated that KR2 has a J intermediate, which is a vibrationally excited state of the primary photoproduct, ${ }^{5,6}$ and a $\mathrm{K}$ intermediate, which is the vibrationally relaxed primary photoproduct, ${ }^{6,7}$ as other microbial rhodopsins. ${ }^{8}$ Furthermore, flash photolysis experiments showed that an $\mathrm{L} / \mathrm{M}$ intermediate, which is an equilibrium of $\mathrm{L}$ and $\mathrm{M}$ states, is formed in $\sim 20 \mu \mathrm{s} .{ }^{1}$ According to the crystal structure of the $\mathrm{L}$ intermediate of bacteriorhodopsin, the retinal Schiff base is located closer to the counterions in the $\mathrm{L}$ intermediate than in the $\mathrm{K}$ intermediate..$^{9,10}$ The $\mathrm{M}$ state is a typical intermediate that appears after proton transfer from the retinal Schiff base to a counterion. ${ }^{11}$ Subsequently, an O intermediate, in which a proton is reconstituted to the 13 -cis retinal, ${ }^{11}$ is formed in $\sim 1 \mathrm{~ms}$, and goes back to the initial ground state in a few tens of milliseconds. ${ }^{1}$ According to the crystal structures reported by Kato et al. in $2015,{ }^{2}$ it is suggested that Asp116 flips in the M-like 

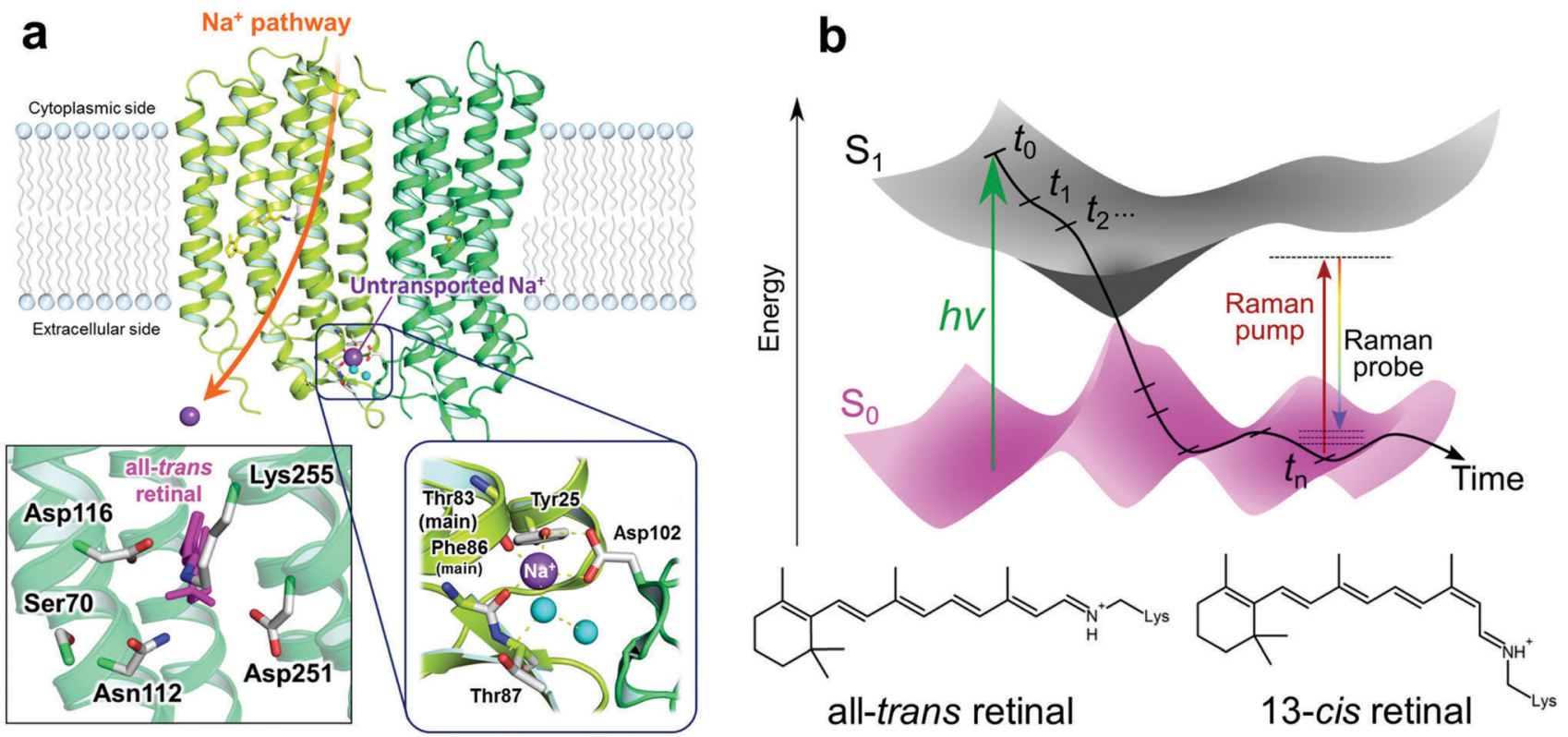

Fig. 1 The extracellular $\mathrm{Na}^{+}$binding site of KR2 and a schematic diagram of transient stimulated Raman spectroscopy. (a) Crystal structure of KR2 ${ }^{4}$ (PDB ID: 4XTN) and close-up of the retinal binding pocket and the $\mathrm{Na}^{+}$binding site on the extracellular side. The $\mathrm{Na}^{+}$binding site consists of Tyr25 (on TM1), Thr87 and main chain carbonyl groups of Thr83 and Phe86 (on TM2) of the same protomer, Asp102 (on the loop between TM2 and TM3) of the neighboring protomer and two water molecules. (b) Schematic diagram of transient stimulated Raman spectroscopy. A narrowband Raman pump $(\sim 800 \mathrm{~nm})$ and a broadband Raman probe $(\sim 840-960 \mathrm{~nm})$ are applied simultaneously after a time delay. The time delay was varied from -50 ps to $110 \mu$ s with the shortest step of $50 \mathrm{fs}$.

intermediate with the proton transfer from the retinal Schiff base to Asp116, resulting in opening the pathway of $\mathrm{Na}^{+}$transport near the retinal Schiff base. Thus, it is considered that the retinal plays important roles not only in triggering the photoreaction, but also in the $\mathrm{Na}^{+}$pump function. Therefore, observation of the retinal dynamics in earlier time regions than the $\mathrm{L} / \mathrm{M}$ formation is essential to clarify the molecular mechanism of KR2.

Femtosecond stimulated Raman spectroscopy (FSRS) is a time-resolved vibrational spectroscopic technique that features high temporal $(\sim 100 \mathrm{fs})$ and spectral $\left(<10 \mathrm{~cm}^{-1}\right)$ resolutions (Fig. 1b) $)^{12}$ and broadband spectral detection. When the wavelength of the Raman pump is tuned resonant or pre-resonant for the chromophore, chromophore-specific observation is possible without disturbance by signals from the protein backbone and side chains. As a major drawback of FSRS, it suffers from notorious problems that arise from unpredictable high-amplitude baselines. ${ }^{13}$ Recently, we solved these baseline problems with a spectral watermarking approach, involving shaping of the Raman pump in combination with wavelet transform of pseudorandomly generated watermarks, which enables us to automatically eliminate the baseline and minimize the fixed-pattern noise of the CCD detectors. Furthermore, we have extended the FSRS technique with widerange continuous time delays from $\sim 10^{-14} \mathrm{~s}$ to $\sim 10^{-4} \mathrm{~s}$ utilizing synchronized ultrafast Ti:sapphire amplifiers ${ }^{15}$ which enables us to investigate chromophore dynamics from femtoseconds to submilliseconds in detail in a single experiment. We call this technique Transient Stimulated Raman Spectroscopy (TSRS).

Here we present femto- to submillisecond TSRS results on WT KR2 in $\mathrm{NaCl}\left(\mathrm{Na}^{+}\right.$bound, $\mathrm{Na}^{+}$transport), the D102N mutant in $\mathrm{NaCl}\left(\mathrm{Na}^{+}\right.$unbound, $\mathrm{Na}^{+}$transport) and WT KR2 in $\mathrm{CsCl}\left(\mathrm{Na}^{+}\right.$ unbound, $\mathrm{H}^{+}$transport). With the spectral data, we will discuss the retinal dynamics on the excited state and the photoproduct states up to the L/M-intermediate formation. Moreover, we will show that the extracellular $\mathrm{Na}^{+}$binding significantly affects the ps- $\mu$ s retinal chromophore dynamics, and that the identity of the transported ion, $\mathrm{H}^{+}$or $\mathrm{Na}^{+}$, does not affect the ps- $\mu$ s reaction.

\section{Results}

\section{Femto- to submillisecond transient absorption spectroscopy}

For reference, we first applied femto- to submillisecond UV-vis transient absorption spectroscopy to KR2. The time-resolved data were globally analyzed in terms of a kinetic model of sequentially interconverting species with increasing time constants. ${ }^{16}$ Fig. 2 shows steady state absorption spectra and globally fitted transient absorption spectra (Evolution-Associated Difference Spectra, EADS) of WT KR2 in NaCl up to $150 \mu \mathrm{s}$ (Decay-Associated Difference Spectra, DADS, are shown in Fig. S1, ESI $\dagger$ ). Nine components were required to adequately represent the transient absorption data with the following time constants: $20 \mathrm{fs}, 170 \mathrm{fs}$, $420 \mathrm{fs}, 2.4 \mathrm{ps}, 29 \mathrm{ps}, 6.5 \mathrm{~ns}, 370 \mathrm{~ns}, 18 \mu \mathrm{s}$ and infinity. Fig. S2-S4 (ESI $\dagger$ ) show raw data along with the fit results. The decay components up to $100 \mathrm{ps}$ are consistent with recently reported femtosecond pump-probe spectroscopy of KR $2,{ }^{8}$ and the $18 \mu \mathrm{s}$ decay component corresponds to the $\sim 26 \mu$ s component in the flashphotolysis experiment. ${ }^{1}$ The first component of the global fitting was created immediately after photon absorption, 

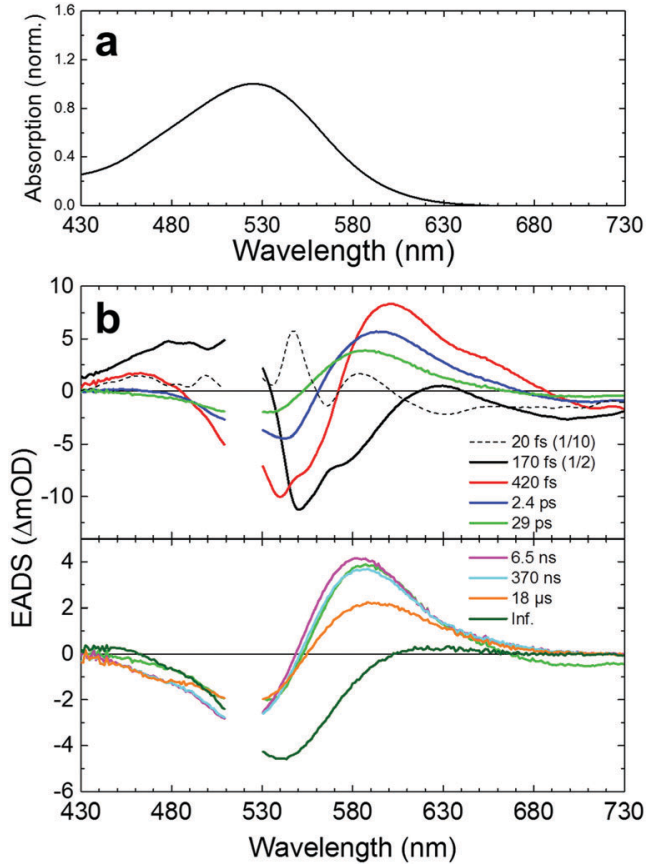

Fig. 2 Steady state and transient absorption results of WT KR2 in $\mathrm{NaCl}$. (a) Steady state absorption of WT KR2 in NaCl. (b) Globally fitted evolutionassociated difference spectra (EADS) of WT KR2 in $\mathrm{NaCl}$. The first five (top) and the following four (bottom) time components are shown separately. The EADS are cut between $510-530 \mathrm{~nm}$ because of the strong pump light scattering. The components of $20 \mathrm{fs}$ and $170 \mathrm{fs}$ are scaled down by a factor of 10 and 2, respectively. The 29 ps component is shown on both of the top and the bottom figures for reference.

possibly mixed as a precursor state and a coherent artifact from overlapping of pump and probe pulses. On the second component, typical peaks assigned as exited state absorption (ESA, at $\sim 480 \mathrm{~nm}$ and at $\sim 630 \mathrm{~nm}$ ), ground state bleach (GSB, at $\sim 550 \mathrm{~nm}$ ) and stimulated emission (SE, at $>\sim 680 \mathrm{~nm}$ ) were seen. Those peaks apparently overlap each other, influencing the intensity and the peak positions. The positive signals on the EADS consist of ESA and/or photoproducts. Transient absorption spectra of KR2/D102N in $\mathrm{NaCl}$ and $\mathrm{WT} \mathrm{KR} 2$ in $\mathrm{CsCl}$ were also globally fitted with nine exponential components (Fig. S5 and S6, ESI $\dagger$ ) as well as WT KR2 in NaCl. No significant difference was seen on the transient absorption spectra of the three different sample conditions within the spectral resolution. The time constants of the transient absorption spectra are shown in Table S1 (ESI $\dagger$ ).

\section{Femto- to submillisecond transient stimulated Raman spectroscopy}

Fig. 3a shows baseline-free stimulated Raman spectra on the ground state with all-trans retinal in WT KR2 in $\mathrm{NaCl}, \mathrm{KR} 2 /$ $\mathrm{D} 102 \mathrm{~N}$ in $\mathrm{NaCl}$ and WT KR2 in CsCl. The ground state Raman spectra were almost identical between WT KR2 in $\mathrm{NaCl}$ and $\mathrm{CsCl}$, and $\mathrm{KR} 2 / \mathrm{D} 102 \mathrm{~N}$ mutant. The $1532 \mathrm{~cm}^{-1}$ band is assigned as the $\mathrm{C}=\mathrm{C}$ ethylenic stretching mode. ${ }^{17,18}$ The $1169 \mathrm{~cm}^{-1}$ and $1201 \mathrm{~cm}^{-1}$ bands are assigned as C-C stretching mode. ${ }^{18,19}$ Typical hydrogen out-of-plane (HOOP) vibrational modes were seen at 831 and $884 \mathrm{~cm}^{-1}$, and methyl rocking mode was

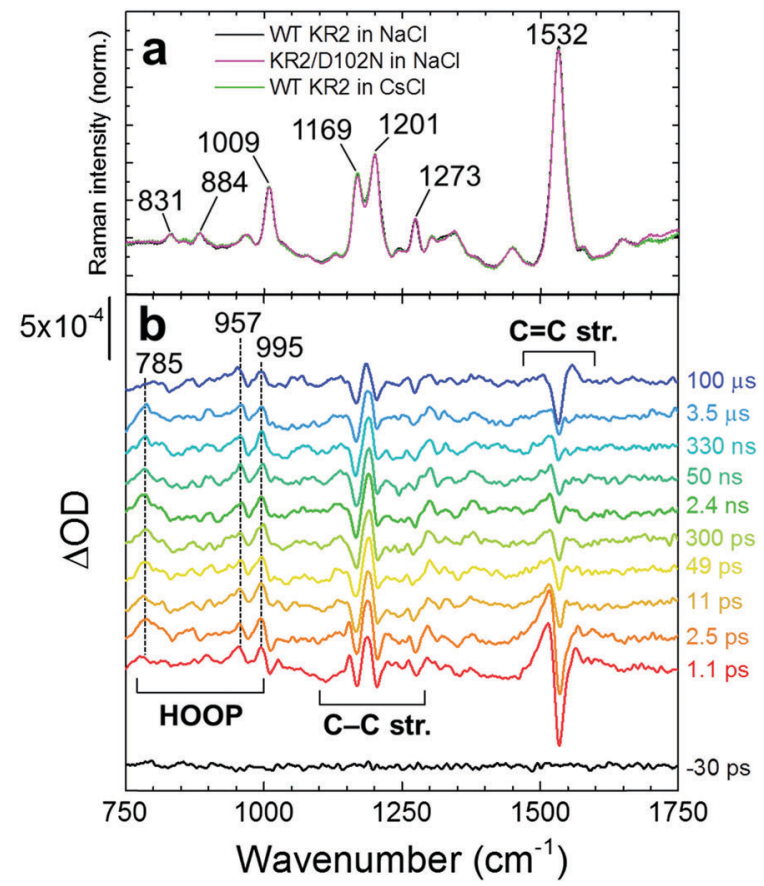

Fig. 3 Baseline-free stimulated Raman spectra of WT KR2 in $\mathrm{NaCl}$. (a) Ground state stimulated Raman spectra of WT KR2 in $\mathrm{NaCl}$ (black), $\mathrm{KR} 2 / \mathrm{D} 102 \mathrm{~N}$ in $\mathrm{NaCl}$ (magenta) and $\mathrm{WT} \mathrm{KR} 2$ in $\mathrm{CsCl}$ (light green). (b) Selected transient stimulated Raman spectra in WT KR2 in NaCl.

observed at $1009 \mathrm{~cm}^{-1} \cdot{ }^{18,19}$ In Fig. 3b, transient stimulated Raman spectra of WT KR2 in $\mathrm{NaCl}$ at selected time delays are shown, represented as the difference stimulated Raman spectrum between actinic pulse on minus actinic pulse off. We prefer to show the TSRS spectra as such difference spectra instead of conventional reconstructed all-positive Raman spectra, because for the latter a certain amplitude of the ground-state stimulated Raman spectrum has to be added, which requires visual inspection and therefore introduces a bias. In total, TSRS spectra at 96 delays were recorded. Positive and negative transient Raman signals were seen in the HOOP $\left(\sim 800-1000 \mathrm{~cm}^{-1}\right)$, the C-C stretching $(\sim 1150-$ $\left.1250 \mathrm{~cm}^{-1}\right)$ and the $\mathrm{C}=\mathrm{C}$ stretching $\left(\sim 1510-1560 \mathrm{~cm}^{-1}\right)$ regions, whose evolution will be shown with globally fitted data in detail.

The transient stimulated Raman spectra of WT KR2 were globally fitted with seven time components: $20 \mathrm{fs}, 260 \mathrm{fs}, 3.4 \mathrm{ps}$, 22 ps, 25 ns, $21 \mu$ s and infinity. Fig. S7-S9 (ESI $\dagger$ ) show the raw, wavelet-processed TSRS data along with the fitting results. The 20 fs component is probably a coherent artifact with the threepulse mixing and is not further considered. Fig. 4 shows EADS of the transient Raman spectra including HOOP, C-C stretching and $\mathrm{C}=\mathrm{C}$ stretching regions (The DADS are shown in Fig. S10, ESI $\dagger$ ). The negative signals show GSB, and the positive signals show Raman spectra on the excited state and/or photoproduct states. In the transient absorption measurement, SE was seen in the near-infrared region at very early delay times in KR2 (Fig. S11, $\mathrm{ESI} \dagger$ ), so Raman initiated by nonlinear emission (RINE) ${ }^{20}$ signals that decayed mostly with 260 fs were observed on the excited state because of the $800 \mathrm{~nm}$ Raman pump pulses. The difference in number of fitting components in the transient Raman (seven) 

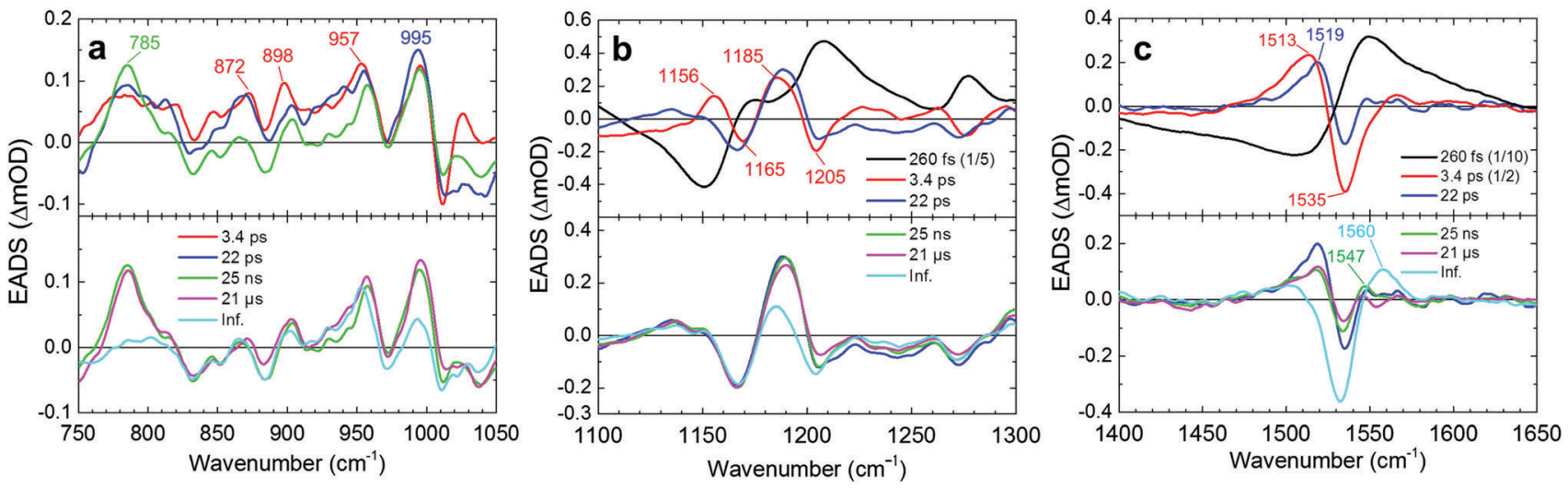

Fig. 4 Globally fitted stimulated Raman spectra (EADS) of WT KR2 in NaCl. The signal evolves as 260 fs (black), 3.4 ps (red), 22 ps (blue), 25 ns (green), $21 \mu$ s (magenta) and an infinite time constant (cyan) in order. (a) EADS at $750-1050 \mathrm{~cm}^{-1}$ including the HOOP region. The 260 fs component is not shown. The first three (top) and the following two (bottom) time components are shown separately. The 25 ns component is shown on both of the top and the bottom figures for reference. EADS at (b) $1100-1300 \mathrm{~cm}^{-1}$ including the $C-C$ stretching region and (c) $1400-1650 \mathrm{~cm}^{-1}$ including the $C=C$ stretching region. The $260 \mathrm{fs}$ (in b and c) and 3.4 ps (in c) components are scaled down. The first three (top) and the following three (bottom) time components are shown separately. The 22 ps component is shown on both of the top and the bottom figures for reference.

and the transient absorption (nine) spectra probably results from a lower signal-to-noise ratio in the former. Significant spectral shifts on transient Raman signals were seen in the C-C stretching region (Fig. 4b). Positive signals at 1156 and $1185 \mathrm{~cm}^{-1}$ appeared in $260 \mathrm{fs},{ }^{19}$ while ground state bleach signals at $1169 \mathrm{~cm}^{-1}$ and $1205 \mathrm{~cm}^{-1}$ were seen. Similar peaks at $1194 \mathrm{~cm}^{-1}$ (positive), 1167 and $1201 \mathrm{~cm}^{-1}$ (negative) reported in $77 \mathrm{~K}$ FT-IR experiments, ${ }^{19}$ indicating those peaks are derived from the 13-cis retinal isomer in the $\mathrm{K}$ intermediate.

In the $\mathrm{C}=\mathrm{C}$ stretching region, a negative signal at around $1535 \mathrm{~cm}^{-1}$ was seen in the transient Raman spectra after $260 \mathrm{fs}$, which was continuous up to $100 \mu$ s (Fig. 4c). On the other hand, a positive signal at $1513 \mathrm{~cm}^{-1}$ appeared in $260 \mathrm{fs}$. Importantly, in $3.4 \mathrm{ps}$, the peak at $1513 \mathrm{~cm}^{-1}$ was blue-shifted to $1519 \mathrm{~cm}^{-1}$. The third spectrum has a lifetime of 22 ps (blue spectra in Fig. 4) and is almost identical to the $77 \mathrm{~K}$ FT-IR experiments. ${ }^{19}$ In $22 \mathrm{ps}$, the peak intensity at $1519 \mathrm{~cm}^{-1}$ decreased by a factor of 2 , while a peak at $1547 \mathrm{~cm}^{-1}$ appeared. In $25 \mathrm{~ns}$, the peak intensity at $1519 \mathrm{~cm}^{-1}$ was slightly increased, and that at $1547 \mathrm{~cm}^{-1}$ was decreased. In $21 \mu \mathrm{s}$, a positive peak at $\sim 1560 \mathrm{~cm}^{-1}$ appeared. Simultaneously with the $1560 \mathrm{~cm}^{-1}$ signal appearance, the positive peak at $1519 \mathrm{~cm}^{-1}$ completely decayed while the signal at $1547 \mathrm{~cm}^{-1}$ remained.

Clearly, positive difference Raman signals were seen in the HOOP region $\left(\sim 800-1000 \mathrm{~cm}^{-1}\right)$; e.g. at 785, 872, 898, 957 and $995 \mathrm{~cm}^{-1}$ after $260 \mathrm{fs}$, while no clear negative bands appeared in the HOOP region. In $22 \mathrm{ps,} \mathrm{HOOP} \mathrm{signals} \mathrm{at} \sim 830-930 \mathrm{~cm}^{-1}$ dropped, while some strong positive signals remained up to $25 \mathrm{~ns}$. Significant signal decrease of other peaks such as at 785 and $995 \mathrm{~cm}^{-1}$ was seen in $21 \mu \mathrm{s}$.

Similarly to WT KR2 in $\mathrm{NaCl}$, transient Raman spectra of KR2/D102N in $\mathrm{NaCl}$ and KR2 WT in CsCl were globally fitted with seven exponential components (Fig. S12, S13 and Table S2, ESI $\dagger$ ). Transient Raman spectra from 1.0 ps to $100 \mu$ s are shown in Fig. 5a-c. Fig. 5d shows transient Raman spectra of WT KR2 in $\mathrm{NaCl}, \mathrm{KR} 2 / \mathrm{D} 102 \mathrm{~N}$ in $\mathrm{NaCl}$ and $\mathrm{WT} \mathrm{KR} 2$ in $\mathrm{CsCl}$ at $4.5 \mathrm{ps}$,
$50 \mathrm{ps}, 480 \mathrm{~ns}$ and $100 \mu \mathrm{s}$. The spectra at 4.5 ps were similar between the three different samples. However, different features were seen at $50 \mathrm{ps}$ and at $480 \mathrm{~ns}$ between WT KR2 in $\mathrm{NaCl}$ and the other two samples in the $\mathrm{C}=\mathrm{C}$ stretching mode region; the $\sim 1547 \mathrm{~cm}^{-1}$ peak gained amplitude as compared to the $\sim 1519 \mathrm{~cm}^{-1}$ peak in KR2/D102N in NaCl and WT KR2 in CsCl, while the $\sim 1519 \mathrm{~cm}^{-1}$ peak was more significant in WT KR2 in $\mathrm{NaCl}$. At $100 \mu \mathrm{s}$, the $\sim 1560 \mathrm{~cm}^{-1}$ signal appeared in all of the three samples while the $\sim 1547 \mathrm{~cm}^{-1}$ signal remained in KR2/ D102N in NaCl and WT KR2 in CsCl. Notably, the characteristics of the transient Raman spectra of KR2/D102N in NaCl and WT KR2 in $\mathrm{CsCl}$ were very similar up to $100 \mu \mathrm{s}$. In Fig. $5 \mathrm{e}-\mathrm{g}$, the evolution of signals at $1519 \mathrm{~cm}^{-1}, 1547 \mathrm{~cm}^{-1}$ and $1560 \mathrm{~cm}^{-1}$ are shown. In $\sim 20 \mathrm{ps}$, the $1547 \mathrm{~cm}^{-1}$ signal rose above that of $1519 \mathrm{~cm}^{-1}$ in KR2/D102N in $\mathrm{NaCl}$ and WT KR2 in $\mathrm{CsCl}$, differently from WT KR2 in NaCl. In $\sim 60 \mathrm{~ns}$, the signal at $1519 \mathrm{~cm}^{-1}$ slightly rose again, while the $1547 \mathrm{~cm}^{-1}$ signal decayed in the three different samples. In $\sim 20 \mu$ s, a blue-shifted peak appeared at $1560 \mathrm{~cm}^{-1}$ as in WT KR2 in NaCl, but the $1547 \mathrm{~cm}^{-1}$ signal remained at a higher amplitude in KR2/D102N in $\mathrm{NaCl}$ and WT KR2 in CsCl than in WT KR2 in NaCl.

\section{Discussion}

With femtosecond-to-picosecond time resolved stimulated Raman spectra, we discuss the excited-state dynamics and the retinal isomerization. The 260 fs decay of the RINE signals, which result from non-linear emission on the excited state, ${ }^{20}$ implies excited-state population decays mostly in 260 fs. Since typical vibrational peaks from 13-cis retinal $\left(1185 \mathrm{~cm}^{-1}\right)^{19}$ appeared in $260 \mathrm{fs}$ with the ground state bleach at 1165 and $1205 \mathrm{~cm}^{-1}$, it is clearly shown that the first isomerized $\mathrm{J}$ intermediate is formed in $260 \mathrm{fs}$. The $\sim 200 \mathrm{fs}$ J-intermediate formation can be also characterized by transient absorption spectra (Fig. 2b); in $\sim 200 \mathrm{fs,}$ the red-shifted species at $602 \mathrm{~nm}$ appeared with the decay of the 

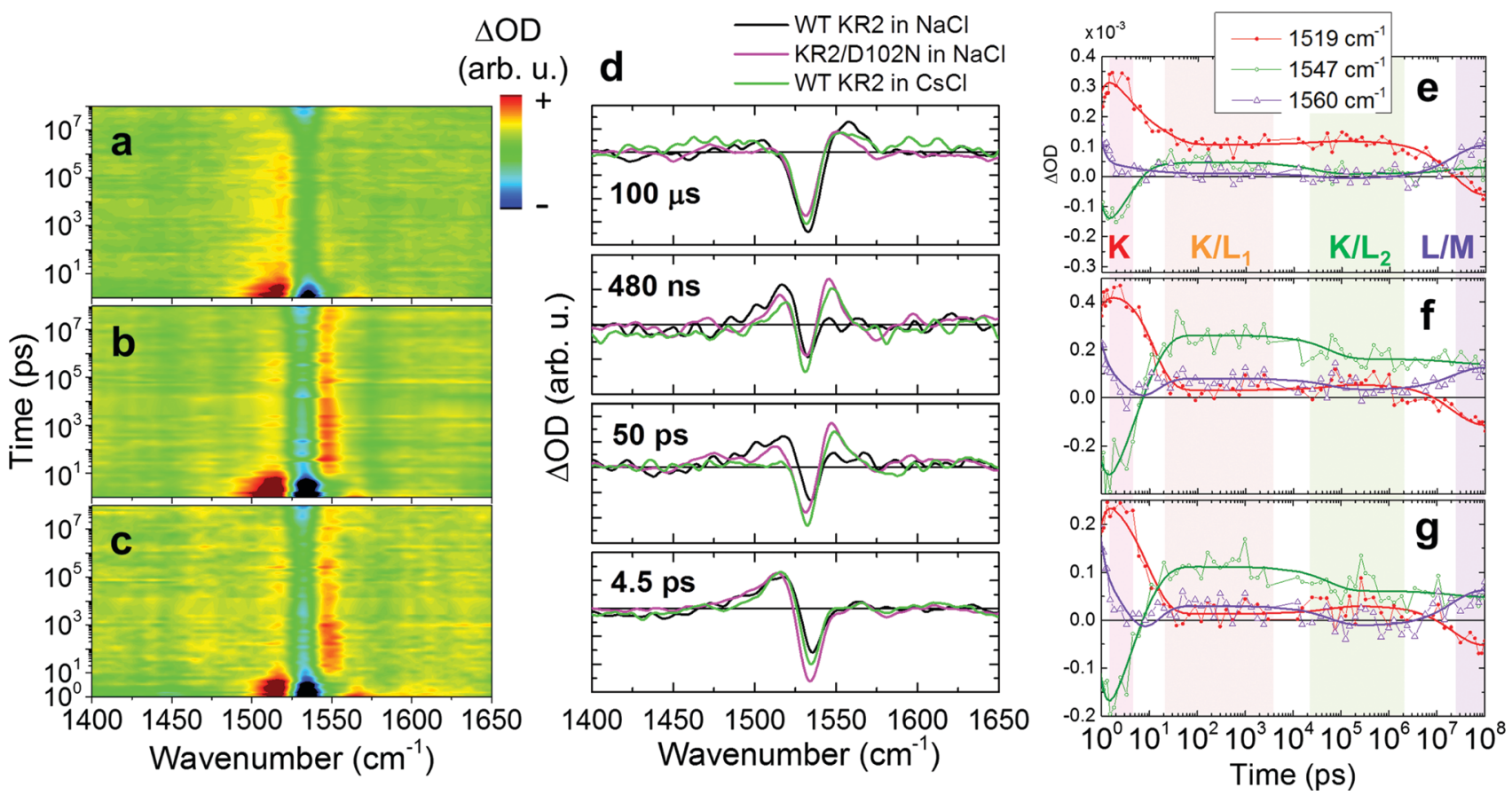

Fig. 5 Transient stimulated Raman spectra in the $\mathrm{C}=\mathrm{C}$ stretching region in WT KR2 in $\mathrm{NaCl}$ KR2/D102N in NaCl and WT KR2 in CsCl. Two-dimensional false color representation of transient stimulated Raman spectra of (a) WT KR2 in NaCl, (b) KR2/D102N in NaCl and (c) WT KR2 in CsCl in the spectral region of 1400-1650 $\mathrm{cm}^{-1}$. The difference Raman signals are color-coded as red for positive, green for zero and blue for negative. (d) Normalized transient Raman spectra of WT KR2 in $\mathrm{NaCl}$ (black), KR2/D102N in NaCl (magenta) and WT KR2 in CsCl (light green) at 4.5 ps, 50 ps, $480 \mathrm{~ns}$ and $100 \mu$ s. Time traces of stimulated Raman signals at $1519 \mathrm{~cm}^{-1}$ (red closed dots with a red fitting line), $1547 \mathrm{~cm}^{-1}$ (open green dots with a green fitting line) and $1560 \mathrm{~cm}^{-1}$ (open purple triangles with a purple fitting line) in (e) WT KR2 in $\mathrm{NaCl}$, (f) KR2/D102N in NaCl and (g) WT KR2 in CsCl.

stimulated emission (Fig. 2b, Fig. S1 and S2, ESI †), which indicates the first photoproduct $\mathrm{J}$ intermediate is formed in $\sim 200 \mathrm{fs}^{8}{ }^{8}$ Because the frequency of the $\mathrm{C}=\mathrm{C}$ stretching mode is correlated with the retinal absorption energy, it is considered that the red-shifted $\mathrm{C}=\mathrm{C}$ stretching mode $\left(1513 \mathrm{~cm}^{-1}\right)$ corresponds to the J intermediate (Fig. 4c). Notably, the appearance of the positive HOOP signals after the J-intermediate formation indicates the 13-cis retinal is markedly distorted compared to the all-trans retinal, as previously shown in bacteriorhodopsin. ${ }^{21}$

Here we discuss the J-to-K state transition, which is interpreted as a vibrational cooling on the primary photoproduct state. When the system relaxes to the bottom of the potential energy well, the energy gap for the electronic transition to higher electronic states becomes larger, which results in the blue shift of the absorption. Thus, observation of the blue shift is useful to identify the time scale of the J-to-K transition. On the transient absorption spectra in Fig. 2, the blue shift was observed in two time constants: 420 fs and 2.4 ps. However, owing to the broad photoproduct absorption peaks and the overlap with the negative signals from the ground state bleach and the stimulated emission, it is difficult to decide which time component is involved in the J-to-K transition. On the other hand, the Raman spectra have narrower peaks, which make it easier to identify the time scale of the K-intermediate formation. We observe a $6 \mathrm{~cm}^{-1}$ blue shift on the $\mathrm{C}=\mathrm{C}$ stretching region with $\sim 3$ ps in EADS (Fig. 4c) and the time trace of the peak position (Fig. S14, ESI $\dagger$ ), indicating that the J-to-K transition occurs on that timescale. A similar blue-shifted $\mathrm{C}=\mathrm{C}$ stretching mode in J-to-K transition was seen in bacteriorhodopsin. ${ }^{21}$

Next, we discuss the retinal dynamics of KR2 on the photoproduct intermediates after the K-intermediate formation. The EADS of the transient Raman spectra in the $\mathrm{C}=\mathrm{C}$ stretching region (1510-1560 $\mathrm{cm}^{-1}$ ) indicate that there are four different intermediate photoproduct states up to $100 \mu \mathrm{s}$ : evolving with $22 \mathrm{ps}, 25 \mathrm{~ns}, 21 \mu \mathrm{s}$ and longer than $100 \mu \mathrm{s}$ (infinity) (Fig. 4). The blue-shifted $\mathrm{C}=\mathrm{C}$ Raman signal at $\sim 1547 \mathrm{~cm}^{-1}$ that rose in $22 \mathrm{ps}$ is assigned as the L state, which is a typical blue-shifted absorption species in microbial rhodopsins. ${ }^{9,11,22}$ With the L-state formation, the HOOP signals at $\sim 830-930 \mathrm{~cm}^{-1}$ dropped (Fig. 4a), which suggests that the retinal chromophore structurally relaxes, but this may also result from slightly reduced resonance conditions of $\mathrm{L}$ with respect to $\mathrm{K}$. In channelrhodopsin, the $\mathrm{L}$ state showed significant HOOP intensity in time-resolved infrared spectroscopy. ${ }^{23}$ Significantly, the L-state signal $\left(1547 \mathrm{~cm}^{-1}\right)$ rose in $22 \mathrm{ps}$ while the K-state signal $\left(1519 \mathrm{~cm}^{-1}\right)$ did not completely disappear (Fig. 4c, green line and Fig. 5e). The coexistence of the positive peaks at $1519 \mathrm{~cm}^{-1}$ and $1547 \mathrm{~cm}^{-1}$ indicates that KR2 forms distinct K/L equilibrium states between $\sim 20$ ps and $\sim 20 \mu \mathrm{s}$. The two distinct newly found $\mathrm{K} / \mathrm{L}$ equilibrium intermediates are described as $\mathrm{K} / \mathrm{L}_{1}$ (formed in $22 \mathrm{ps,} \mathrm{Fig.} \mathrm{4c,} \mathrm{green}$ line) and $\mathrm{K} / \mathrm{L}_{2}$ (formed in $25 \mathrm{~ns}$, Fig. 4c, magenta line) intermediates. In $25 \mathrm{~ns}$, the $1519 \mathrm{~cm}^{-1}$ signal slightly rose again while the peak intensity at $1547 \mathrm{~cm}^{-1}$ decreased (Fig. 4c), which implies the equilibrium of $\mathrm{K} / \mathrm{L}$ shifts back to $\mathrm{K}$ in the $\mathrm{K} / \mathrm{L}_{2}$ intermediate 


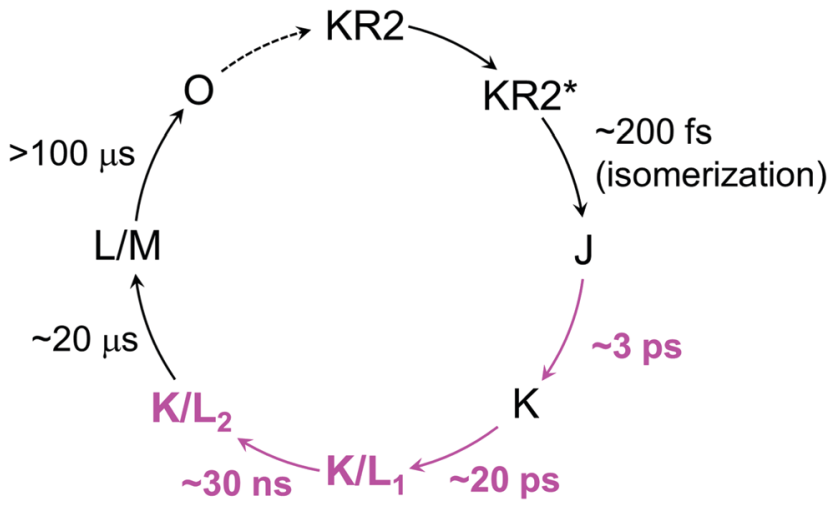

Fig. 6 A new photocycle model of WT KR2 in $\mathrm{NaCl}$. The newly identified intermediates and time components by this work are high-lighted as magenta. The O-intermediate formation and decay have previously been proposed. ${ }^{1,8}$

before the formation of the following $\mathrm{L} / \mathrm{M}$ intermediate. Even though the L-state structure of KR2 is unsolved, it can be suggested that the retinal Schiff base is oriented to the counterions with a closer distance on the $\mathrm{L}$ state, similar to bacteriorhodopsin.,10 Because the $\mathrm{K} / \mathrm{L}$ equilibrium slightly shifts back to $\mathrm{K}$ again in $\sim 30 \mathrm{~ns}$, the distance between the retinal Schiff base and the counterions might become longer before the M-state formation.

The blue-shifted $1560 \mathrm{~cm}^{-1}$ band, which forms in $\sim 20 \mu \mathrm{s}$ can be assigned as the $\mathrm{M}$ state (Fig. 4c, cyan line). The mixture of the positive peaks at $1547 \mathrm{~cm}^{-1}$ and $1560 \mathrm{~cm}^{-1}$ indicates the L state coexists with the $\mathrm{M}$ state, forming an $\mathrm{L} / \mathrm{M}$ intermediate in $\sim 20 \mu$ s as seen in the flash photolysis experiments ${ }^{1}$ and our transient absorption experiments (Fig. 2). Significant decrease of the HOOP signals at 785 and $995 \mathrm{~cm}^{-1}$ in $21 \mu \mathrm{s}$ implies that the twist of the retinal is relaxed with the formation of the $\mathbf{M}$ state as seen in bacteriorhodopsin. ${ }^{24}$ From the overall discussion above, a new photocycle model from femtoseconds to submilliseconds is proposed in Fig. 6.

We now compare the retinal dynamics of WT KR2 in $\mathrm{NaCl}$ ( $\mathrm{Na}^{+}$bound, $\mathrm{Na}^{+}$transport), KR2/D102N in $\mathrm{NaCl}\left(\mathrm{Na}^{+}\right.$unbound, $\mathrm{Na}^{+}$transport) and WT KR2 in $\mathrm{CsCl}\left(\mathrm{Na}^{+}\right.$unbound, $\mathrm{H}^{+}$transport). A similar photocycle up to $100 \mu \mathrm{s}$ in Fig. 6 can be applied to the three different sample conditions, albeit with important differences as outlined below. At $4.5 \mathrm{ps}, 50 \mathrm{ps}, 480 \mathrm{~ns}$ and $100 \mu \mathrm{s}$, the retinal of $\mathrm{KR} 2$ is in the $\mathrm{K}, \mathrm{K} / \mathrm{L}_{1}, \mathrm{~K} / \mathrm{L}_{2}$ and $\mathrm{L} / \mathrm{M}$ intermediate, respectively (Fig. $5 \mathrm{~d}$ ). The ground state stimulated Raman spectra of WT KR2 in NaCl, KR2/D102N in NaCl and WT $\mathrm{KR} 2$ in $\mathrm{CsCl}$ were almost identical as seen in Fig. 3a. Moreover, no significant difference was seen in transient Raman spectra at $4.5 \mathrm{ps}$ in the three different conditions as seen in $77 \mathrm{~K}$ FT-IR experiments. ${ }^{19}$ These results imply that the molecular structure of the all-trans retinal and the 13-cis retinal in the $\mathrm{K}$ intermediate is identical regardless of the $\mathrm{Na}^{+}$binding on the extracellular side and whether $\mathrm{Na}^{+}$or $\mathrm{H}^{+}$is transported.

In the $\mathrm{K} / \mathrm{L}_{1}$ intermediate, clear differences between the three different samples were observed in the TSRS experiments: the L state signal $\left(1547 \mathrm{~cm}^{-1}\right)$ is dominant as compared to the $\mathrm{K}$ state signal $\left(1519 \mathrm{~cm}^{-1}\right)$ in $\mathrm{KR} 2 / \mathrm{D} 102 \mathrm{~N}$ in $\mathrm{NaCl}$ and WT KR2 in $\mathrm{CsCl}$, while the $\mathrm{K}$ state is more significant than the L state in WT KR2 in $\mathrm{NaCl}$, as clearly seen in the false color time-wavenumber plots of Fig. 5a-c, and the time-resolved Raman spectra of Fig. 5 d. These observations imply that the equilibrium in the $\mathrm{K} / \mathrm{L}_{1}$ intermediate shifts to $\mathrm{L}$ in the absence of $\mathrm{Na}^{+}$binding at the extracellular side. In the $\mathrm{K} / \mathrm{L}_{2}$ intermediate, the equilibrium slightly shifts to $\mathrm{K}$ as compared to in $\mathrm{K} / \mathrm{L}_{1}$ intermediate, although the $\mathrm{L}$ state is still dominant in the absence of $\mathrm{Na}^{+}$ binding on the extracellular side. The shift of the K/L equilibrium is clearly seen in the time traces of $1519 \mathrm{~cm}^{-1}$ and $1547 \mathrm{~cm}^{-1}$ signals in Fig. 5e-g. Furthermore, in the $\mathrm{L} / \mathrm{M}$ intermediate at $100 \mu \mathrm{s}$, the M-state peak at $1560 \mathrm{~cm}^{-1}$ has lower amplitude in the D102N mutant and WT KR2 in CsCl (Fig. 5d). This indicates that the equilibrium shifts to $\mathrm{L}$ in the $\mathrm{L} / \mathrm{M}$ intermediate in the absence of the extracellular $\mathrm{Na}^{+}$binding. Possibly, the distance between the retinal Schiff base and the counterions is different with and without the extracellular $\mathrm{Na}^{+}$binding, which makes the difference in the $\mathrm{K} / \mathrm{L} / \mathrm{M}$ equilibrium (Fig. 7). Notably, in the case where $\mathrm{Na}^{+}$is unbound to $\mathrm{KR} 2$, the $\mathrm{K} / \mathrm{L} / \mathrm{M}$ equilibrium state is very similar regardless of which ion, $\mathrm{Na}^{+}$or $\mathrm{H}^{+}$, is transported.

A protomer of the pentameric KR2 has two different $\mathrm{Na}^{+}$ binding sites; (i) bound to Tyr25 (on TM1) and Thr87 main chain carbonyl groups of Thr83 and Phe86 (on TM2), and (ii) bound to Asp102 (on TM2-TM3 loop) (Fig. 1a). ${ }^{4}$ On the other hand, monomeric KR2 has only a $\mathrm{Na}^{+}$binding site (i), near Tyr25 and Thr87, lacking the $\mathrm{Na}^{+}$binding to Asp102 on the TM2-TM3 loop. ${ }^{4}$ Between the monomeric and pentameric forms, significant structural differences were seen in the first half of TM3, while no notable difference was observed in other regions (Fig. S15, ESI $\dagger$ ). ${ }^{4}$ Possibly, $\mathrm{Na}^{+}$binding to Asp102 affects the structure of the half of TM3 even though the structural difference might be just from difference of the oligomeric states. The structural difference on the half of TM3 is perhaps involved in the $\mathrm{K} / \mathrm{L} / \mathrm{M}$ equilibrium states, although it does not

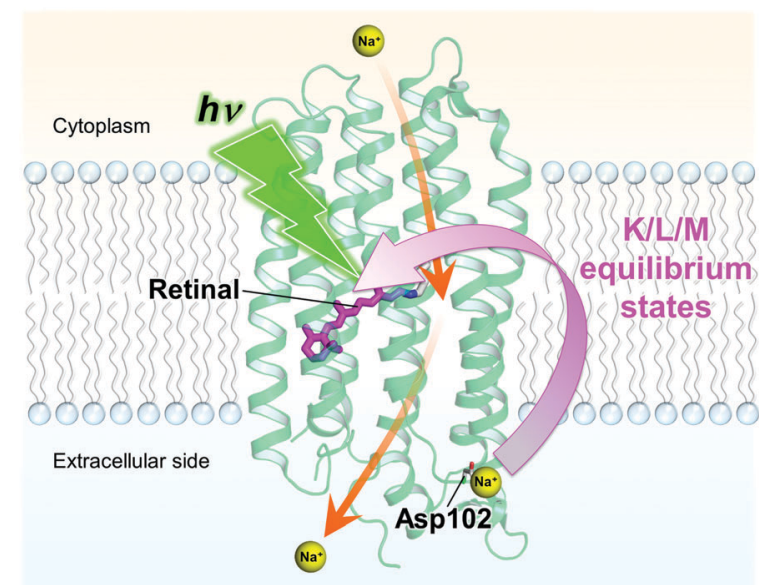

Fig. 7 Proposed model of the extracellular $\mathrm{Na}^{+}$binding that affects the primary dynamics of retinal between picoseconds and microseconds. The $\mathrm{Na}^{+}$ion bound on the extracellular side affects the $\mathrm{K} / \mathrm{L}$ and $\mathrm{L} / \mathrm{M}$ equilibrium states of the retinal. The protein structure is based on Gushchin et al. (PDB ID: 4 XTN). The proposed $\mathrm{Na}^{+}$pumping pathway ${ }^{1}$ is drawn with orange arrows. 
affect the retinal structure on the steady state and the $\mathrm{K}$ intermediate. However, to fully understand how $\mathrm{Na}^{+}$influences the ps- $\mu$ s retinal dynamics in $\mathrm{K} / \mathrm{L}_{1}, \mathrm{~K} / \mathrm{L}_{2}$ and $\mathrm{L} / \mathrm{M}$ intermediates, tracing backbone transitions in those time scales with further time-resolved experiments is required.

\section{Conclusion}

We investigated the photocycle of sodium ion pump rhodopsin KR2 by applying the watermarked femto- to submillisecond transient stimulated Raman spectroscopy technique. By considering the transient Raman spectra on hydrogen-out-of-plane (HOOP), $\mathrm{C}-\mathrm{C}$ stretching and $\mathrm{C}=\mathrm{C}$ stretching modes, we propose a new photocycle model of KR2, evolving to J (formed in $\sim 200 \mathrm{fs}$ ) $\rightarrow$ $\mathrm{K}(\sim 3 \mathrm{ps}) \rightarrow \mathrm{K} / \mathrm{L}_{1}(\sim 20 \mathrm{ps}) \rightarrow \mathrm{K} / \mathrm{L}_{2}(\sim 30 \mathrm{~ns}) \rightarrow \mathrm{L} / \mathrm{M}(\sim 20 \mu \mathrm{s})$. We also observed that the retinal conformation and the dynamics in the three probed conditions were almost identical in the ground state and the $\mathrm{K}$ intermediate. However, significant differences were observed in $\mathrm{K} / \mathrm{L}_{1}, \mathrm{~K} / \mathrm{L}_{2}$ and $\mathrm{L} / \mathrm{M}$ intermediates, as the $\mathrm{K} / \mathrm{L}$ and $\mathrm{L} / \mathrm{M}$ equilibrium shifts to $\mathrm{L}$ in the absence of extracellular bound $\mathrm{Na}^{+}$. By our research, it was revealed for the first time that the extracellular $\mathrm{Na}^{+}$binding plays an important role on the primary photoreactions from picoseconds to microseconds, while the identity of the transported ion, $\mathrm{Na}^{+}$or $\mathrm{H}^{+}$, does not affect the photocycle.

\section{Methods}

\section{Protein expression and purification}

The protein samples of WT KR2 and D102N mutant were expressed in E. coli (C41(DE3) strain) and purified as previously reported. ${ }^{2}$ The purified proteins were solubilized in $1.0 \%$ $n$-dodecyl- $\beta$-D-maltoside (DDM), $100 \mathrm{mM} \mathrm{NaCl,} 50 \mathrm{mM}$ Tris-HCl ( $\mathrm{pH}$ 8.0). For the spectroscopic measurement in $\mathrm{CsCl}$ solution, the solvent was exchanged to that containing $100 \mathrm{mM} \mathrm{CsCl}$ instead of $\mathrm{NaCl}$. The proteins were concentrated to $200 \mu \mathrm{M}$ (5.0 $\mathrm{mg} \mathrm{mL} \mathrm{m}^{-1}$ ) for transient absorption experiments, and to $2.0 \mathrm{mM}\left(50 \mathrm{mg} \mathrm{mL}{ }^{-1}\right)$ for stimulated Raman experiments, with using a centrifugal filter device (Millipore $30 \mathrm{kDa} \mathrm{Mw}$ cutoff). The samples were filled in a homemade sample holder that has two $2 \mathrm{~mm}$-thick $\mathrm{CaF}_{2}$ plates. The sample thickness was set as $400 \mu \mathrm{m}$ for transient absorption experiments and $200 \mu \mathrm{m}$ for stimulated Raman experiments with an appropriate sample spacer. The sample holder was set on a Lissajous scanner that ensures sample refreshment between laser shots with a time interval of 60 seconds between successive exposures to the laser pulses. ${ }^{25}$

\section{Transient absorption spectroscopy}

Transient absorption measurements were performed with a femtosecond-to-submillisecond pump-probe setup as reported previously. ${ }^{15}$ A sapphire plate was used for supercontinuum white light generation, and a selected wavelength region; 430$730 \mathrm{~nm}$ or $720-940 \mathrm{~nm}$, wase detected by the photodiode array. The time delay was varied up to $150 \mu \mathrm{s}$ at 164 data points with the minimum temporal step of $50 \mathrm{fs}$. The diameters of the pump and the probe beams at the sample position were $\sim 200 \mu \mathrm{m}$ and $\sim 70 \mu \mathrm{m}$, respectively. The wavelength of the pump beam was centered at $520 \mathrm{~nm}$, and the power was attenuated to $\sim 500 \mathrm{~nJ}$. The instrumental response function was $\sim 80 \mathrm{fs}$, estimated from global analysis.

\section{Baseline-free transient stimulated Raman spectroscopy}

Femtosecond to submillisecond time-resolved stimulated Raman experiments were performed with the watermarked baseline-free stimulated Raman setup reported previously. ${ }^{14}$ Raman pump $(800 \mathrm{~nm}, \sim 20 \mu \mathrm{J})$ and Raman probe $(\sim 840-960 \mathrm{~nm})$ were spatiotemporally overlapped at the sample position with the diameter of $\sim 100 \mu \mathrm{m}$. Actinic pump $(\sim 520 \mathrm{~nm}, \sim 600 \mathrm{~nJ})$ was focused on the protein sample to a diameter of $\sim 150 \mu \mathrm{m}$ with a time delay from -50 ps to $110 \mu$ s at 96 data points (logarithmically spaced after $2 \mathrm{ps}$ ), generated by an optical delay and an electronic delay as reported previously. ${ }^{15}$ Raman pumps pass through a specially-designed chopper blade for watermarking approach, ${ }^{14}$ which produces 14 Raman pump sequences whose wavelengths are slightly shifted each other. ${ }^{14}$ As a result, 14 different stimulated Raman experiments are effectively performed simultaneously, which makes the baseline-free watermarking approach possible. The sample exposure time to the beams was $\sim 1$ hour in total for each time-resolved stimulated Raman experiment.

\section{Global analysis methodology}

Global analysis was performed for the transient absorption spectra and the transient stimulated Raman spectra using the Glotaran program. ${ }^{15,26}$ With global analysis, all wavelengths/ wavenumbers were analyzed simultaneously with a set of common time constants. ${ }^{16}$ A kinetic model was applied consisting of sequentially interconverting, evolution-associated difference spectra (EADS), i.e. $1 \rightarrow 2 \rightarrow 3 \rightarrow \cdots$ in which the arrows indicate successive monoexponential decays of a time constant, which can be regarded as the lifetime of each EADS. ${ }^{16}$ The first EADS corresponds to the difference spectrum at time zero. The first EADS evolves into the second EADS with time constant $\tau_{1}$, which in turn evolves into the third EADS with time constant $\tau_{2}$, etc. The procedure clearly visualizes the evolution of the intermediate states of the protein. ${ }^{27}$ Decayassociated difference spectra (DADS) indicate the spectral changes with parallel decay channels and independent decay time constants. It is important to note that the parallel and the sequential analysis are mathematically equivalent and yield identical time constants. ${ }^{28}$ The standard errors in the time constants were less than $5 \%$ for the transient absorption and less than $10 \%$ for the transient stimulated Raman results. ${ }^{26,27}$

\section{Author contributions}

K. I. and Y. K. expressed and purified the samples; Y. H. performed transient absorption experiments; Y. H., K. I. and M. K. performed transient stimulated Raman experiments; Y. H. analyzed the data; Y. H. and K. I. wrote the manuscript, edited by H. K. and J. T. M. K.; H. K. and J. T. M. K. supervised the project. 


\section{Competing financial interests}

The authors declare no competing financial interests.

\section{Acknowledgements}

Y. H., M. K. and J. T. M. K. were supported by the Chemical Sciences Council of the Netherlands Organization for Scientific Research (NWO) through a VICI grant to J. T. M. K., a VENI grant to M. K. and a Middelgroot investment grant to J. T. M. K. This work was also supported by JSPS KAKENHI Grant to K. I. (26708001, 26115706, 26620005) and H. K. (25104009, 15H02391).

\section{References}

1 K. Inoue, H. Ono, R. Abe-Yoshizumi, S. Yoshizawa, H. Ito, K. Kogure and H. Kandori, Nat. Commun., 2013, 4, 1678.

2 H. E. Kato, K. Inoue, R. Abe-Yoshizumi, Y. Kato, H. Ono, M. Konno, S. Hososhima, T. Ishizuka, M. R. Hoque, H. Kunitomo, J. Ito, S. Yoshizawa, K. Yamashita, M. Takemoto, T. Nishizawa, R. Taniguchi, K. Kogure, A. D. Maturana, Y. Iino, H. Yawo, R. Ishitani, H. Kandori and O. Nureki, Nature, 2015, 521, 48-53.

3 I. Gushchin, V. Shevchenko, V. Polovinkin, V. Borshchevskiy, P. Buslaev, E. Bamberg and V. Gordeliy, FEBS J., 2016, 283, 1232-1238.

4 I. Gushchin, V. Shevchenko, V. Polovinkin, K. Kovalev, A. Alekseev, E. Round, V. Borshchevskiy, T. Balandin, A. Popov, T. Gensch, C. Fahlke, C. Bamann, D. Willbold, G. Buldt, E. Bamberg and V. Gordeliy, Nat. Struct. Mol. Biol., 2015, 22, 390-395.

5 R. A. Mathies, C. H. Brito Cruz, W. T. Pollard and C. V. Shank, Science, 1988, 240, 777-779.

6 D. Xu, C. Martin and K. Schulten, Biophys. J., 1996, 70, 453-460.

7 K. C. Hasson, F. Gai and P. A. Anfinrud, Proc. Natl. Acad. Sci. U. S. A., 1996, 93, 15124-15129.

8 S. Tahara, S. Takeuchi, R. Abe-Yoshizumi, K. Inoue, H. Ohtani, H. Kandori and T. Tahara, J. Phys. Chem. Lett., 2015, 6, 4481-4486. 9 J. K. Lanyi and B. Schobert, J. Mol. Biol., 2003, 328, 439-450.

10 T. Kouyama, T. Nishikawa, T. Tokuhisa and H. Okumura, J. Mol. Biol., 2004, 335, 531-546.
11 O. P. Ernst, D. T. Lodowski, M. Elstner, P. Hegemann, L. S. Brown and H. Kandori, Chem. Rev., 2014, 114, 126-163.

12 P. Kukura, D. W. McCamant and R. A. Mathies, Annu. Rev. Phys. Chem., 2007, 58, 461-488.

13 M. Kloz, R. van Grondelle and J. T. M. Kennis, Phys. Chem. Chem. Phys., 2011, 13, 18123-18133.

14 M. Kloz, J. WeiSsenborn, T. Polivka, H. A. Frank and J. T. M. Kennis, Phys. Chem. Chem. Phys., 2016, 18, 14619-14628.

15 J. Ravensbergen, F. F. Abdi, J. H. van Santen, R. N. Frese, B. Dam, R. van de Krol and J. T. M. Kennis, J. Phys. Chem. C, 2014, 118, 27793-27800.

16 I. H. M. van Stokkum, D. S. Larsen and R. van Grondelle, Biochim. Biophys. Acta, Bioenerg., 2004, 1657, 82-104.

17 T. Althaus, W. Eisfeld, R. Lohrmann and M. Stockburger, Isr. J. Chem., 1995, 35, 227-251.

18 S. O. Smith, M. S. Braiman, A. B. Myers, J. A. Pardoen, J. M. L. Courtin, C. Winkel, J. Lugtenburg and R. A. Mathies, J. Am. Chem. Soc., 1987, 109, 3108-3125.

19 H. Ono, K. Inoue, R. Abe-Yoshizumi and H. Kandori, J. Phys. Chem. B, 2014, 118, 4784-4792.

20 D. W. McCamant, P. Kukura and R. A. Mathies, J. Phys. Chem. B, 2005, 109, 10449-10457.

21 S. Shim, J. Dasgupta and R. A. Mathies, J. Am. Chem. Soc., 2009, 131, 7592-7597.

22 L. Zimanyi, J. Saltiel, L. S. Brown and J. K. Lanyi, J. Phys. Chem. A, 2006, 110, 2318-2321.

23 V. A. Lorenz-Fonfria, T. Resler, N. Krause, M. Nack, M. Gossing, G. F. von Mollard, C. Bamann, E. Bamberg, R. Schlesinger and J. Heberle, Proc. Natl. Acad. Sci. U. S. A., 2013, 110, E1273-E1281.

24 J. Sasaki, A. Maeda, C. Kato and H. Hamaguchi, Biochemistry, 1993, 32, 867-871.

25 M. T. Alexandre, T. Domratcheva, C. Bonetti, L. J. van Wilderen, R. van Grondelle, M. L. Groot, K. J. Hellingwerf and J. T. Kennis, Biophys. J., 2009, 97, 227-237.

26 J. J. Snellenburg, S. P. Laptenok, R. Seger, K. M. Mullen and I. H. M. van Stokkum, J. Stat. Softw., 2012, 49, 1-22.

27 J. T. M. Kennis and M. L. Groot, Curr. Opin. Struct. Biol., 2007, 17, 623-630.

28 K. C. Toh, E. A. Stojkovic, I. H. van Stokkum, K. Moffat and J. T. Kennis, Phys. Chem. Chem. Phys., 2011, 13, 11985-11997. 